\title{
Cytological Diagnosis of an Uncommon Cause of Post-Menopausal Vaginitis
}

\author{
Manjari Kishore ${ }^{1}$, Prajwala Gupta ${ }^{2 *}$ and Minakshi Bhardwaj ${ }^{3}$ \\ ${ }^{1}$ Senior Resident, Dept. of Pathology, PGIMER, Dr. RML Hospital, New Delhi \\ ${ }^{2}$ Associate Professor, Dept. of Pathology, PGIMER, Dr. RML Hospital, New Delhi \\ ${ }^{3}$ Professor \& Head, Dept. of Pathology, PGIMER, Dr. RML Hospital, New Delhi
}

\section{Dear Sir,}

Trichomoniasis is an infectious disease caused by the parasite Trichomonas vaginalis. It is a sexually transmitted infection. Majority of patients are asymptomatic; however, symptoms includes itching in the genital area, foul smelling thin vaginal discharge, burning micturition and dyspareunia. ${ }^{[1-4]}$ Complications for symptomatic women include vaginitis, endometritis, infertility and cervical cancer. Complications for symptomatic men include urethritis, prostatitis, epididymitis, and infertility. It is also associated with increased risk of transmission and acquisition of HIV. ${ }^{[4-6]}$ Usually this infection is noted in the reproductive age group. We present a case of trichomonas infection which was incidentally detected in a postmenopausal female with underlying uterine fibroid.

A 55-year-old female presented to hospital with complaints of discharge per vaginum along with pain in lower abdomen for last 3 months. She had attained menopause 2 years back. However, she had irregular episodes of bleeding for last 2-3 months. Her parity was 2 with last child birth 24 years ago following which surgical ligation was done. There was no history of hypertension, tuberculosis and diabetes mellitus.

Per speculum examination showed hypertrophied, inflamed cervix. Her per-vaginal examination revealed anteverted, irregular and mobile uterus. However, transvaginal sonography (TVS) showed presence of single large uterine fibroid in the posterior wall.

Complete blood count and routine biochemical investigations were within normal limit. Cervical Pap smear examination showed presence of dense inflammation along with singly scattered pear-shaped organism, suggestive of Trichomonas vaginalis infection with focal debris [Figure $1 \mathrm{~A}-\mathrm{C}]$. There was no evidence of any intraepithelial lesion or underlying malignancy. A diagnosis of Trichomonial infection was made and the patient was symptomatically managed with antibiotics. Hysterectomy was done in view of large intramural fibroid and irregular vaginal bleeding.
Sir, Trichomonas vaginalis organism resides on squamous epithelium of the urogenital tract. Most people infected with trichomonas vaginalis do not have any symptoms and can remain undetected for years. ${ }^{[3-5]}$ For women there may also be a yellow-green, itchy, frothy, foul-smelling ("fishy" smell) vaginal discharge. In rare cases, lower abdominal pain can occur. Symptoms usually appear within 5 to 28 days of exposure.

The human genital tract is the only reservoir for this species. Trichomonas is transmitted through sexual or genital contact. Trichomonas infection is seen in women during reproductive years but vanishes after menopause. [2-6] The single-celled protozoan produces mechanical stress on host cells and then ingests cell fragments after cell death. Trichomoniasis is associated with increased risk of transmission and infection of HIV. It also causes lysis of epithelial cells and red blood cells in the area leading to genital inflammation and the leakiness.

T.vaginalis has also been reported in the urinary tract, fallopian tubes, and pelvis and may cause pneumonia, bronchitis, and oral lesion. The role of trichomonas infection in causing cervical cancer is unclear, although trichomonas infection may be associated with co-infection with high-risk strains of HPV. ${ }^{[3-6]}$

Trichomoniasis may cause a woman to deliver a low-birthweight or premature infant. ${ }^{[5-6]}$ T. vaginalis infection in males has been found to cause asymptomatic urethritis and prostatitis. The postmenopausal women who suffer from a hormonal deficiency disease, there is gradual atrophy of the genital organs and the vaginal wall becomes thin and smooth with decreased acidity, thereby allowing the organisms to survive and cause vaginitis.

Vaginal trichomoniasis is diagnosed either by the observation of motile protozoa on a wet mount of vaginal discharge, or by culturing the organism. Some infections are also diagnosed by Cervicovaginal Pap smear cytology. $[5-6]$ 


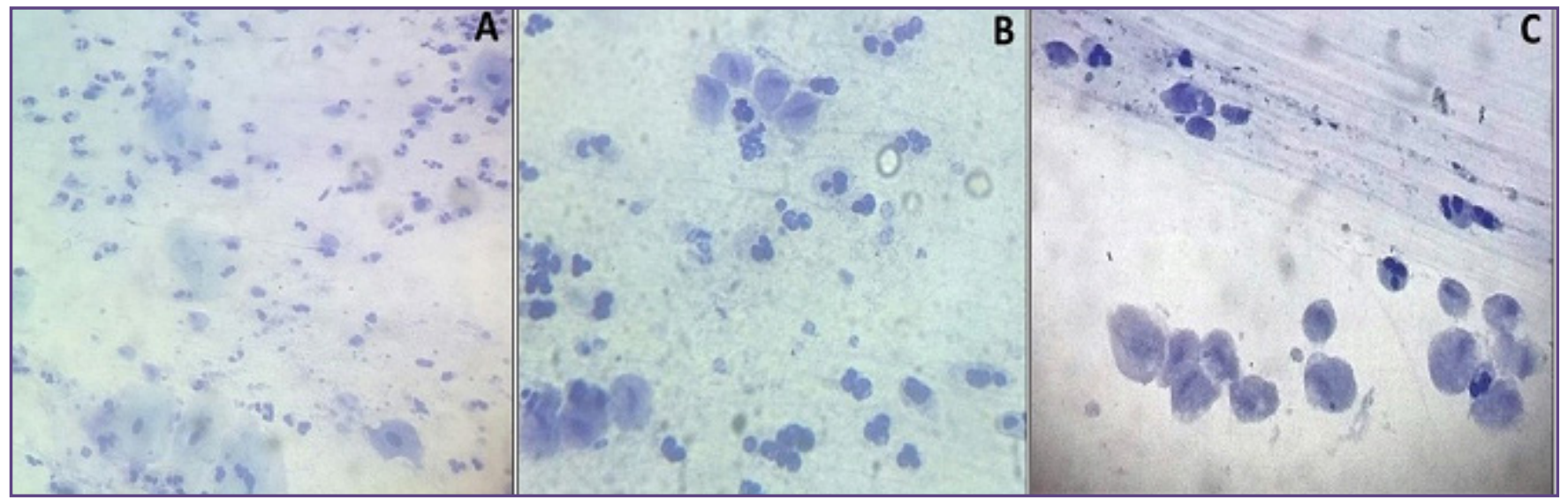

Fig. 1A-C: A- Smear showing dense acute inflammation with interspersed mature squamous cells, few with koilocytic change(marked with red arrow) [Pap, 200X]; B\&C- Many trophozoites of Trichomonas vaginalis organism are noted with neutrophilic collection [Pap, B-200X; C-400X].

Sir, the present case reiterates the importance of a simple, age old test, i.e. cervical Pap smear examination, in early and reliable detection of Trichomoniasis and thereby facilitates patient management.

\section{Reference}

1. Sood, S., Butel, J. Morsa,S. 'In Pouch Trichomonis Vaginalis culture for detection of Trichomonis vaginalis ''Indian J. Med Res 2007;125(10):567-571.

2. Ryan, F.J., Ray, G.G. Sherries Medical Microbiology" 4thed, Mc Grow Hill, 2001.

3. Arroyo R.L. Ladif, A.S. Stevens, C.E., Alexander, W.J. 'Molecular basis of epithelial cell recognition by
T.vaginalis" Molecular Microbiology 2006;6(7): 853862.

4. Mavedzeng, Sue Napierata, Van derpol, Cheng, Helen, Ramjee, Kelly, Elizabeth "' Epidemiological Synergy of Trichomonas vaginalis and HIV in Zimbabwean and South African Women" Sexually Transmitted Disease 2010;37 (7): 460-6.

5. Krieger JN, Verdon M, Siegel N, et al.Risk assessment and laboratory diagnosis of trichomoniasis in men. J Infect Dis 1992;166:1362-6.

6. Mullah R. Summaiya W., Kosambiya J., Shethwala F. Nimished C. "'Sexually transmitted infections and reproductive tract infections in female sex workers." IJPM 2009;52(2):198-9.

*Corresponding author:

Dr. Prajwala Gupta, Room No.302, 3rd floor, OPD building, Dr RML Hospital, Baba Khadak Singh Maarg, New Delhi-110001

Phone: +91 991036227

Email: prajwala2000@yahoo.com

Financial or other Competing Interests: None. 